Original Research Article

\title{
Study of knowledge, attitude and practice of self medication among health care workers at MC Gann Teaching District hospital of Shivamogga, India
}

\author{
Vedavathi Hanumaiah, Harini Manjunath*
}

Department of Pharmacology, Shivamogga Institute of Medical Sciences Shimoga, Karnataka, India

Received: 02 April 2018

Accepted: 25 April 2018

*Correspondence to:

Dr. Harini Manjunath,

Email: drharini.g@gmail.com

Copyright: (C) the author(s), publisher and licensee Medip Academy. This is an openaccess article distributed under the terms of the Creative Commons Attribution NonCommercial License, which permits unrestricted noncommercial use, distribution, and reproduction in any medium, provided the original work is properly cited.

\begin{abstract}
Background: Healthcare workers gain adequate knowledge related to medications used in treating illness from their work experiences which influences self medication practices.

Methods: The present study was conducted in $\mathrm{N}=150$ healthcare workers, divided into 2 groups with group I (nursing staff) and group II (paramedical staff) with 75 participants in each group. Data related to self medication was obtained from a pretested validated semi structured questionnaire either in Kannada or English. The responses were compared between each group with chi square test. $P$ value $\leq 0.05$ was considered significant. All statistical analysis was conducted with SPSS 16.

Results: The mean age (mean $\pm \mathrm{SD}$ ) of the participants in group I and group II is $31.79 \pm 8.309$ and $34.15( \pm 8.168)$ respectively with $p=0.081$. The prevalence of self medication was $100 \%$ in both the groups. Both the groups knowledge related to the definition of self medication was similar (group I $63(84.0 \%)$ and group II $62(82.7 \%) \mathrm{p}=0.900)$. Group I believes that self medication is entirely safe compared to group II which was statistically significant (group I $66(88.7 \%$ ) and group II $46(61.3 \%) \mathrm{p}=0.029)$. Most common drugs used for self medication was NSAIDS (non steroidal anti-inflammatory drugs) in both the groups being 75 $(100 \%)$. Antibiotics was used by $26(2.66 \%)$ in group I and $14(18.66 \%)$ in group II.
\end{abstract}

Conclusions: Self medication practice is highly prevalent in the healthcare workers, who also influence the other populations to practice self medication. Practicing responsible self medication is more appreciable.

Keywords: Healthcare workers, Health, Self medication, OTC

\section{INTRODUCTION}

Health is an important factor need for better survival. ${ }^{1}$ WHO defines "self care" as "the ability of individuals, families and communities to promote health, prevent disease, maintain health, and to cope with illness and disability with or without the support of a health-care provider". ${ }^{2}$ Self care involves activities and practices that are engaged on a regular or time basis to maintain health, relax mind, reduce stress, engage practices to prevent diseases and treatment of minor ailments with home remedies or medication ${ }^{2}$. There is an increase practice of self care due to several factors like; socioeconomic factors; lifestyle; easy access to drugs; the increased potential to manage certain illnesses through self-care; public health and environmental factors; greater availability of medicinal products; and demographic and epidemiological factors. ${ }^{3}$

Self medication is defined as obtaining and consuming drugs without the advice of a physician either for diagnosis, prescription or surveillance of treatment. It includes procuring medicines without a prescription, self decision to reuse old prescriptions to purchase the same 
medicines prescribed for illness without consulting the doctor, using medicines as advised by relatives or friends and using medication due to influence of media. ${ }^{4}$ Self medication is an important component of self care and most of the drugs used for self medication are the OTC (over the counter) drugs. ${ }^{4}$ OTC drugs are medications which can be procured without the need for a physicians prescription. Not all drugs used in self medication are OTC drugs. 5,6 WHO advices to follow a responsible self medication which requires knowledge regarding the health status and about medication or treatment with which it would be advantageous in maintaining health. Products used in responsible self medication must provide information regarding: administration of medicines; effects and possible side-effects; monitoring of effects and side effects;possible interactions; precautions and warnings; duration of use; and when to seek professional advice., ${ }^{7,8}$

Healthcare workers play an important role in health management. They are the people who engage in actions with the primary intent to enhance health. They function in supporting several departments of the health departments such as surgery, medicine, dentistry, radiology, ophthalmology, research etc. Nursing and other paramedical staff (OT (operation theatre) technician, optician, lab assistant, statistician etc) are important healthcare workers in the hospital who have adequate knowledge about the medications. ${ }^{9}$ Pharmacology is a part of the syllabus to the nursing, pharmacist and other paramedical staff such as OT technician, optician etc who are trained adequately to use medications under the physicians supervision to the patients. Whereas the other paramedical staff without the knowledge of pharmacology due to the nature of their work practice self medications by observing the drugs prescribed by physicians, availability of wide number of generic medications available in the hospitals, ease of procuring the medications from the working hospitals and are also aware of the indications and dose of the medications to be used. ${ }^{10}$ Thus knowledge of health and medications plays a vital role in self medication. ${ }^{10}$

Self medication practices is highly prevalent all over world with estimated prevalence of $37 \%$ in urban population and $17 \%$ in rural population in India, $68 \%$ in Europe and African countries being 40.7-81.8\%. ${ }^{11-13}$ The prevalence of self medication practices among healthcare workers to be high as $52.1 \%$ in Nigeria. ${ }^{14}$ As the healthcare workers gain adequate knowledge of medications they would influence the other population in practicing the self medication which could have benefits as well as harmful effects. This study was conducted with the objective of evaluating the knowledge, attitude and practices among healthcare workers working at Mc Gann teaching district hospital Shimoga, a tertiary hospital.

\section{METHODS}

After obtaining permission from the institutional Ethics Committee, this prospective cross sectional study was conducted among healthcare workers working at Mc Gann District Teaching Hospital Shimoga within a period of 6 months from june 2016 to October 2017. Total of 150 respondent participants with minimum 1 year of work experience were recruited in the study.

The participants were divided into two groups with 75 participants each. Group I included nursing staff who had completed either of the nursing course such as- BSc nursing, Diploma in nursing, GNM and ANM. Group II included the paramedical staff who had completed a paramedical course either diploma or certificate course such as- DOT (diploma in ophthalmic technology), COTT (certificate course of operation theatre technology), pharmacist, DMXT (Diploma in medical $\mathrm{X}$ ray technology) etc other than nursing staff.

Knowledge attitude and practice was evaluated by a semi structured validated pretested questionnaire to the participants in their understandable language either, in English or in Kannada. The semi structured questionnaire included two parts. Part I consisted of the demographic data (age, gender, place, education station, working position and working experience). Part II consisted of total 30 questions regarding self medication with 10 questions related to knowledge, attitude and practice each. The participants were instructed to answer all the questions provided with their fullest knowledge.

Informed consent was obtained formed all the participants. All the categorical variables were described in term of frequencies and compared between the groups with Chi square test. age of participants was assessed in terms of mean and standard deviation (SD) and was compared with student $t$ test. All the data were entered in Microsoft excel 2007 and statistical assessment was conducted with SPSS 16. A $p$ value with $\leq 0.05$ was considered to be significant.

\section{RESULTS}

\section{Demographic data}

The mean age (mean \pm SD) of the participants in group I and group II are $31.79 \pm 8.309$ and $34.15( \pm 8.168)$ respectively with no statistical difference $\mathrm{p}=0.081$. Female participants were dominant in both the groups with $70(93.3 \%)$ in group I and $62(82.7 \%)$ in group II. Compared to group I 5 (6.7\%), group II 13 (17.3\%) had more number of male participants which was statistically significant $\mathrm{p}=0.044$ (Table 1$)$.

\section{Assessment of knowledge of self medication among participants}

Both the groups knowledge regarding the definition of self medication was almost the same (group I 63 (84.0\%) and group II $62(82.7 \%) \mathrm{p}=0.900)$ and the knowledge pertaining to the diagnosis of self medication was also similar in both the groups (group I $65(86.7 \%)$ and group II $64(85.3 \%) \mathrm{p}=0.456)$. Group II obtained the information 
regarding the medications/ drug used for self medication from media 9books, television, magazine, newspaper) and internet more than Group I which was statistically significant (group I $12(16.0 \%)$ and group II 29 (38.7\%) $\mathrm{p}=0.002)$ and (group I $8(10.7 \%)$ and group II $22(29.3 \%)$ $\mathrm{p}=8.167$ ). Group I knew the correct full form of OTC (over the counter) drugs compared to group II which was statistically significant (group I $58(77.30 \%)$ and group II
$37(49.3 \%) \mathrm{p}=0.001)$. Group II knew better that ayurvedic drugs also come under OTC drugs (group I 16 (21.3\%) and group II $30(40.0 \%) \mathrm{p}=0.012)$. Group I gained knowledge regarding practicing self medication by observing the drug prescribed by the physician more compared to group II (group I $35(46.7 \%)$ and group II $22(29.3 \%) \mathrm{p}=0.029)$ which was statistically significant (Table 2 ).

Table 1: Demographic data.

\begin{tabular}{|c|c|c|c|c|}
\hline Demographic variable & Group I $(n=75)$ & Group II $(n=75)$ & $\mathrm{X}^{2}(\mathrm{df})$ & P value \\
\hline Age $($ Mean \pm SD) & $31.79 \pm 8.30$ & $34.15 \pm 8.16$ & - & 0.081 \\
\hline \multicolumn{5}{|l|}{ Gender } \\
\hline Male & $5(6.7 \%)$ & $13(17.3 \%)$ & $4.04(1)$ & $0.044^{*}$ \\
\hline Female & $70(93.3 \%)$ & $62(82.7 \%)$ & & \\
\hline \multicolumn{5}{|l|}{ Background (n\%) } \\
\hline Rural & $50(66.67 \%)$ & $52(69.33 \%)$ & $0.123(1)$ & 0.726 \\
\hline Urban & $25(33.33 \%)$ & $23(30.67)$ & & \\
\hline \multicolumn{5}{|l|}{ Marital status (n\%) } \\
\hline Married & $62(82.67 \%)$ & $68(90.67 \%)$ & $2.07(1)$ & 0.152 \\
\hline Unmarried & $13(17.33 \%)$ & $7(9.33 \%)$ & & \\
\hline \multicolumn{5}{|c|}{ Year of work experience (n\%) } \\
\hline $1-5 \mathrm{yr}$ & $29(38.66 \%)$ & $17(22.67 \%)$ & $5.90(4)$ & 0.207 \\
\hline $6-10 y r$ & $16(21.33 \%)$ & $24(32.00 \%)$ & & \\
\hline $11-15 y r$ & $13(17.33 \%)$ & $11(14.66 \%)$ & & \\
\hline $16-20 \mathrm{yr}$ & $6(8.0 \%)$ & $7(9.33 \%)$ & & \\
\hline$>20 \mathrm{yr}$ & $11(14.66 \%)$ & $16(21.33 \%)$ & & \\
\hline
\end{tabular}

Table 2: Participants knowledge related to self medication.

\begin{tabular}{|c|c|c|c|c|}
\hline Questions & $\begin{array}{l}\text { Group I } \\
(n=75)\end{array}$ & $\begin{array}{l}\text { Group II } \\
(n=75)\end{array}$ & $\mathbf{X}^{2}(\mathbf{d f})$ & P value \\
\hline 1. Self medication is taking medication without any medical supervision & $63(84.0 \%)$ & $62(82.7 \%)$ & $0.585(1)$ & 0.900 \\
\hline 2.In self medication diagnosis is done by the individual himself & $65(86.7 \%)$ & $64(85.3 \%)$ & $2.608(3)$ & 0.456 \\
\hline \multicolumn{5}{|l|}{ 3.Information about self medication was obtained from } \\
\hline a) Pharmacist & $10(13.3 \%)$ & $15(20.0 \%)$ & $1.200(1)$ & 0.273 \\
\hline b) Individual himself & $20(26.7 \%)$ & $18(24.0 \%)$ & $0.1414(1)$ & 0.707 \\
\hline c) Family members/friends/ colleagues & $33(44.0 \%)$ & $24(32.0 \%)$ & $2.292(1)$ & 0.130 \\
\hline d) Media (television, radio, magazine, newspaper) & $12(16.0 \%)$ & $29(38.7 \%)$ & $9.7(1)$ & $0.002^{*}$ \\
\hline e) Books & $11(14.7 \%)$ & $18(24.0 \%)$ & $2.095(1)$ & 0.148 \\
\hline f) Internet & $8(10.7 \%)$ & $22(29.3 \%)$ & $8.167(1)$ & $0.004 *$ \\
\hline 4. For self medication prescription is necessary & $44(58.7 \%)$ & $48(64.00 \%)$ & $0.450(1)$ & 0.502 \\
\hline 5.OTC drugs stands for & $58(77.3 \%)$ & $37(49.3 \%)$ & $12.660(1)$ & $0.001 *$ \\
\hline $\begin{array}{l}\text { 6. OTC drugs are procured or dispensed by the pharmacist without } \\
\text { prescription }\end{array}$ & $51(68.0 \%)$ & $46(61.3 \%)$ & $0.729(1)$ & 0.393 \\
\hline 7. OTC drugs have no schedule & $40(53.3 \%)$ & $29(38.7 \%)$ & $3.247(1)$ & 0.072 \\
\hline 8. OTC drug does not need prescription & $38(40.0 \%)$ & $41(54.7 \%)$ & $3.932(2)$ & 0.140 \\
\hline 9.Ayurvedic drugs are also considered as OTC drugs & $16(21.3 \%)$ & $30(40.0 \%)$ & $12.873(4)$ & $0.012 *$ \\
\hline \multicolumn{5}{|l|}{ 10.How did you gain knowledge regarding practicing self medication } \\
\hline a) Previous consultation & $12(16.0 \%)$ & $21(28.0 \%)$ & $3.147(1)$ & 0.076 \\
\hline b) Drug advertisement & $7(9.3 \%)$ & $11(14.7 \%)$ & $1.010(1)$ & 0.315 \\
\hline c) From relatives/friends & $7(9.3 \%)$ & $16(21.3 \%)$ & $4.160(1)$ & $0.041 *$ \\
\hline d) From literature & $17(22.7 \%)$ & $12(16.0 \%)$ & $1.069(1)$ & 0.301 \\
\hline e) From Practitioner you work under & $19(25.3 \%)$ & $27(36.0 \%)$ & $2.007(1)$ & 0.157 \\
\hline f) By observing the drug prescribed by the practitioner & $35(46.7 \%)$ & $22(29.3 \%)$ & $4.782(1)$ & $0.029 *$ \\
\hline
\end{tabular}

${ }^{*} \mathrm{P}$ value $\leq 0.05$ 


\section{Assessment of attitude of participants towards self medication}

Group I believes that self medication is entirely safe compared to group II which was statistically significant (group I $66(88.7 \%)$ and group II $46(61.3 \%) \mathrm{p}=0.029)$ and few participants $26(34.7 \%)$ in group II alone believes that self medication is not safe always. Group I prefers self medication as it has advantage of being convenient in treating illness when consultation is unavailable/ as an emergency treatment compared to group I being statistically significant (group I $53(70.66 \%)$ and group II
$23(30.66 \%) \mathrm{p}=<0.0001)$. Group I believed improper/ insufficient dosage and frequency of medication used to be the most common disadvantage of self medication (group I $59(78.66 \%)$ and group II $39(52.00 \%) \mathrm{p}=<0.0001)$ were as Group II believed self medication had more chance of side effects or drug interactions (group I 12 (16.00\%) and group II $58(77.33 \%) \mathrm{p}=<0.0001)$. Group II preferred to seek pharmacist guidance compared to group 1 (group I 44 $(58.70 \%)$ and group II $61(81.33 \%) \mathrm{p}=<0.0001)$. Group II preferred to suggest self medication for people without medical knowledge more compared to group I (group I 38 $(58.70 \%)$ and group II $24(32.0 \%) \mathrm{p}=<0.0001)$ (Table 3).

Table 3: participants attitude related towards self medication.

\begin{tabular}{|c|c|c|c|c|}
\hline Questions & $\begin{array}{l}\text { Group I } \\
(n=75)\end{array}$ & $\begin{array}{l}\text { Group II } \\
(\mathrm{n}=75)\end{array}$ & $X^{2}(d f)$ & $P$ value \\
\hline \multicolumn{5}{|l|}{ 1. Self medication is entirely safe. } \\
\hline a) Yes & $66(88.0 \%)$ & $46(61.3 \%)$ & $32.571(2)$ & $0.001 *$ \\
\hline b) No & $9(12 \%)$ & $3(4.00 \%)$ & & \\
\hline c) Not always & 0 & $26(34.7 \%)$ & & \\
\hline \multicolumn{5}{|l|}{ 2.Self medication have advantages: } \\
\hline a) yes & $58(77.33 \%)$ & $52(69.33 \%)$ & $1.227(1)$ & 0.268 \\
\hline b) No & $17(22.66 \%)$ & $23(30.66 \%)$ & & \\
\hline \multicolumn{5}{|l|}{ 3.The advantages of self medication are: } \\
\hline a) Saves time from consulting doctor & $40(53.33 \%)$ & $22(29.33 \%)$ & $8.908(1)$ & $0.003 *$ \\
\hline b) Helps in treating minor ailments & $43(57.33 \%)$ & $28(37.33 \%)$ & $6.017(1)$ & $0.014 *$ \\
\hline c) Saves consultation fees & $51(68.00 \%)$ & $22(29.33 \%)$ & $22.443(1)$ & $<0.0001^{*}$ \\
\hline $\begin{array}{l}\text { d) Convenient in treating illness when consultation is } \\
\text { unavailable/ as an emergency treatment }\end{array}$ & $53(70.66 \%)$ & $23(30.66 \%)$ & $24.00(1)$ & $<0.0001 *$ \\
\hline e) Immediate relief of symptoms & $41(54.66 \%)$ & $27(36.00 \%)$ & $5.273(1)$ & $0.022 *$ \\
\hline \multicolumn{5}{|l|}{ 4. Self medication has disadvantages: } \\
\hline a) Yes & $65(86.66 \%)$ & $57(76.66 \%)$ & $3.339(2)$ & 0.188 \\
\hline b) No & $10(13.33 \%)$ & $18(24.00 \%)$ & & \\
\hline \multicolumn{5}{|l|}{ 5. Disadvantages of self medications are: } \\
\hline a) incorrect self diagnosis & $49(65.33 \%)$ & $22(29.33 \%)$ & $19.49(1)$ & $<0.0001^{*}$ \\
\hline b) Risk of side effects/interactions & $12(16.00 \%)$ & $58(77.33 \%)$ & $56.679(1)$ & $<0.0001^{*}$ \\
\hline c) Improper/insufficient dosage and frequency & $59(78.66 \%)$ & $39(52.00 \%)$ & $11.774(1)$ & $<0.0001^{*}$ \\
\hline d) Incorrect choice of therapy & $30(40.00 \%)$ & $6(8.00 \%)$ & $21.053(1)$ & $<0.0001^{*}$ \\
\hline e) risk of dependence and addiction & $30(40.00 \%)$ & $8(10.66 \%)$ & $17.058(1)$ & $<0.0001^{*}$ \\
\hline f) can lead to toxicity and death & $19(25.33 \%)$ & $16(21.33 \%)$ & $0.335(1)$ & 0.562 \\
\hline \multicolumn{5}{|l|}{ 6.Do you prefer self medication for } \\
\hline a) Common ailments & $73(97.3)$ & $74(98.7 \%)$ & $0.340(1)$ & 0.560 \\
\hline b) Serious conditions & $2(2.7 \%)$ & $1(1.3 \%)$ & & \\
\hline \multicolumn{5}{|c|}{ 7. Would you like to seek pharmacist guidance before self medication } \\
\hline a) Yes & $44(58.7 \%)$ & $61(81.3 \%)$ & $9.175(1)$ & $0.002 *$ \\
\hline b) No & $31(41.3 \%)$ & $14(18.7 \%)$ & & \\
\hline \multicolumn{5}{|c|}{ 8.Would you like to seed physician opinion if symptoms worsen with self medication } \\
\hline a) Yes & $74(98.7 \%)$ & $74(98.7 \%)$ & $0.000(1)$ & 1.000 \\
\hline b) No & $1(1.3 \%)$ & $1(1.3 \%)$ & & \\
\hline \multicolumn{5}{|c|}{ 9. Do you suggest self medication for people without medical knowledge } \\
\hline a) Yes & $38(50.7 \%)$ & $24(32.0 \%)$ & $5.389(1)$ & $0.020 *$ \\
\hline b) No & $37(49.3 \%)$ & $51(68.0 \%)$ & & \\
\hline \multicolumn{5}{|c|}{ 10. Do you prefer to consult the doctor for treating side effects due to self medication } \\
\hline a) Yes & $75(100 \%)$ & $70(93.3 \%)$ & $5.172(1)$ & $0.023 *$ \\
\hline b) No & 0 & $5(6.7 \%)$ & & \\
\hline
\end{tabular}

${ }^{*} \mathrm{P}$ value $\leq 0.05$ 
Table 4: Participants practices related to self medication.

\begin{tabular}{|c|c|c|c|c|}
\hline Questions & $\begin{array}{l}\text { Group I } \\
(\mathrm{n}=75)\end{array}$ & $\begin{array}{l}\text { Group II } \\
(\mathrm{n}=75)\end{array}$ & $\mathbf{X}^{2}(\mathrm{df})$ & P value \\
\hline \multicolumn{5}{|l|}{ 1. Commonly practiced self medication are drugs are } \\
\hline a) injectables & 0 & $9(12.0 \%)$ & $9.574(1)$ & $0.002 *$ \\
\hline b) non injectables & $75(100 \%)$ & $66(88.0 \%)$ & & \\
\hline \multicolumn{5}{|l|}{ 2. Common medical condition for which self medication is practiced } \\
\hline a) Fever & $67(89.3 \%)$ & $63(84.00 \%)$ & $0.923(1)$ & 0.337 \\
\hline b) Cold & $53(70.6 \%)$ & $48(64.0 \%)$ & $0.759(1)$ & 0.384 \\
\hline c) Cough & $33(44.00 \%)$ & $27(36.0 \%)$ & $1.00(1)$ & 0.317 \\
\hline d) Headache & $47(62.66 \%)$ & $35(46.66 \%)$ & $3.874(1)$ & $0.049 *$ \\
\hline e) Musculoskeletal pain & $44(58.66 \%)$ & $27(36.0 \%)$ & $7.729(1)$ & $0.005 *$ \\
\hline f) Diarrhea & $18(24.00 \%)$ & $18(24.0 \%)$ & $0.00(1)$ & 1.00 \\
\hline g) constipation & $1(1.33 \%)$ & $2(2.66 \%)$ & $0.340(1)$ & 0.560 \\
\hline h) Allergic reactions & $11(14.66 \%)$ & $14(18.66 \%)$ & $0.432(1)$ & 0.511 \\
\hline i) Pain abdomen & $37(49.33 \%)$ & $10(13.33 \%)$ & $22.588(1)$ & $<0.0001 *$ \\
\hline j) Supplements & $8(10.66 \%)$ & $9(0.12 \%)$ & $0.066(1)$ & 0.0797 \\
\hline k) Nausea/Vomiting & $17(22.66 \%)$ & $18(24.00 \%)$ & $0.037(1)$ & 0.847 \\
\hline 1) Gastritis & $34(45.33 \%)$ & $16(21.33 \%)$ & $9.720(1)$ & $0.002 *$ \\
\hline m) Infections & $6(8.00 \%)$ & $1(1.33 \%)$ & $3.746(1)$ & 0.053 \\
\hline n) Burns & $2(2.66 \%)$ & $2(2.66 \%)$ & $0.00(1)$ & 1.00 \\
\hline o) Lack of sleep & $3(4.0 \%)$ & $5(6.66 \%)$ & $3.061(1)$ & 0.680 \\
\hline p) Mouth ulcers & $4(5.33 \%)$ & $5(6.66 \%)$ & $0.118(1)$ & 0.731 \\
\hline \multicolumn{5}{|l|}{ 3.Commonly used drugs for self medication } \\
\hline $\begin{array}{l}\text { a) Analgesics: NSAIDs (Paracetamol, Diclofenac, Aceclofenac, } \\
\text { Aspirin + Caffeine, Ibuprofen) }\end{array}$ & $75(100 \%)$ & $75(100 \%)$ & $1.007(1)$ & 0.314 \\
\hline b) Antihistamines (Cetrizine, Chlorphenaramine, Cinnarizine) & $45(60.00 \%)$ & $38(50.66 \%)$ & $1.322(1)$ & 0.250 \\
\hline $\begin{array}{l}\text { c) Gastrointestinal system: Drugs used for gastritis (Ranitidine, } \\
\text { Pantoprazole, Omeprazole, Suralfate, Antacids) }\end{array}$ & $48(68.00 \%)$ & $38(50.66 \%)$ & $2.725(1)$ & 0.099 \\
\hline Antiemetics (Domperidone, Metaclopramide) & $16(21.33 \%)$ & $8(10.66 \%)$ & $3.175(1)$ & 0.075 \\
\hline Drugs for diarrhea (ORS, Loperamide) & $5(6.66 \%)$ & $9(12.00 \%)$ & $1.261(1)$ & 0.262 \\
\hline Drugs for constipation (Lactulose) & $2(2.66 \%)$ & $3(4.00 \%)$ & $0.000(1)$ & 1.00 \\
\hline Drugs for pain abdomen (Mefenamic acid) & $12(16.00 \%)$ & $2(2.66 \%)$ & $7.878(1)$ & $0.005^{*}$ \\
\hline \multicolumn{5}{|l|}{ d)Antimicrobial agents } \\
\hline $\begin{array}{l}\text { Antibiotics (Amoxicillin+clavulinic acid, Cefexime, Cefadroxil, } \\
\text { Ciprofloxacin, Ofloxacin, Azithromycin, Cefotaxime) }\end{array}$ & $26(34.66 \%)$ & $14(18.66 \%)$ & $4.909(1)$ & $0.027 *$ \\
\hline Antihelminthics (Albendazole) & $2(2.66 \%)$ & $3(4.00 \%)$ & $0.207(1)$ & 0.649 \\
\hline Antiprotozoal agents (Metronidazole) & $1(1.33 \%)$ & 0 & $1.007(1)$ & 0.316 \\
\hline \multicolumn{5}{|l|}{ e) Respiratory system } \\
\hline Drugs for cough (syrup- Ascoril, Benadryl, ambrolite) & $12(16.00 \%)$ & $12(16.00 \%)$ & $0.000(1)$ & 1.00 \\
\hline \multicolumn{5}{|l|}{ f) CNS } \\
\hline Drugs for insomnia (Diazepam, Alprazolam) & $1(1.33 \%)$ & $3(4.00 \%)$ & $1.027(1)$ & 0.311 \\
\hline $\begin{array}{l}\text { g) Dermatologics (Calamine lotion, silversulfadiazine cream, } \\
\text { Neomycin powder, cotrimazole powder, Betamethasone cream, } \\
\text { Betadine ointment) }\end{array}$ & $9(12.00 \%)$ & $9(12.00 \%)$ & $0.000(1)$ & 1.00 \\
\hline h) Nutritional Supplements (Calcium, Vitamin) & $14(18.66 \%)$ & $7(9.33 \%)$ & $2.713(1)$ & 0.100 \\
\hline \multicolumn{5}{|l|}{ 4. Do you store drugs used for self medication } \\
\hline a) Yes & $53(70.7 \%)$ & $53(70.7 \%)$ & 0.00 & 1.00 \\
\hline b) No & $22(29.3 \%)$ & $22(29.3 \%)$ & & \\
\hline \multicolumn{5}{|l|}{ 5. Most common drugs used for self medication are: } \\
\hline a) Ayurvedic & $29(38.7 \%)$ & $28(37.3 \%)$ & $0.28(1)$ & 0.866 \\
\hline b) Allopathy & $48(64.0 \%)$ & $42(55.0 \%)$ & $1.00(1)$ & 0.317 \\
\hline c) Homeopathy & $11(14.7 \%)$ & $10(13.3 \%)$ & $0.055(1)$ & 0.814 \\
\hline \multicolumn{5}{|l|}{ 6. Have you ever practiced self medication } \\
\hline a) Yes & $68(90.7 \%)$ & $61(81.3 \%)$ & 2.713(1) & 0.100 \\
\hline
\end{tabular}


Table 4: Continue...

\begin{tabular}{|c|c|c|c|c|}
\hline Questions & $\begin{array}{l}\text { Group I } \\
(\mathrm{n}=75)\end{array}$ & $\begin{array}{l}\text { Group II } \\
(n=75)\end{array}$ & $\mathrm{X}^{2}(\mathrm{df})$ & $P$ value \\
\hline b) No & $7(9.3 \%)$ & $14(18.7 \%)$ & & \\
\hline \multicolumn{5}{|c|}{ 7. Were the symptoms/ illness relieved on self medication } \\
\hline a) Yes & $58(77.3 \%)$ & $57(76.0 \%)$ & $0.037(1)$ & 0.847 \\
\hline b) $\mathrm{No}$ & $17(22.7 \%)$ & $18(24.0 \%)$ & & \\
\hline \multicolumn{5}{|c|}{ 8. Have you experienced side effects after self medication } \\
\hline a) Yes & $17(22.7 \%)$ & $18(24.0 \%)$ & $0.037(1)$ & 0.847 \\
\hline b) No & $58(77.3 \%)$ & $57(76.0 \%)$ & & \\
\hline \multicolumn{5}{|c|}{ 9. Do you practice self medication to treat side effects occurred due to self medication } \\
\hline a) Yes & $10(13.3 \%)$ & $33(30.7 \%)$ & \multirow{2}{*}{$6.566(1)$} & \multirow{2}{*}{$0.010^{*}$} \\
\hline b) No & $65(86.7 \%)$ & $52(69.3 \%)$ & & \\
\hline \multicolumn{5}{|c|}{ 10. Do you read drug information sheet before self medication } \\
\hline a) Yes & $59(78.7 \%)$ & $66(88.0 \%)$ & 2.352(1) & 0.125 \\
\hline b) No & $16(21.3 \%)$ & $9(12.0 \%)$ & & \\
\hline
\end{tabular}

\section{Assessment of practice of self medication among participants}

There were $68(90.7 \%)$ in group I and $61(81.3 \%)$ practiced self medication $(\mathrm{p}=0.100)$ and in group II alone $9(12.0 \%)$ participants preferred injectables for self medication $(\mathrm{p}=0.002)$. The most common illness (Figure 1) for which self medication was practiced was fever in both the groups I [group I $67(89.30 \%)$ and group II $63(84.00 \%)$ p=0.337]. Most common drugs used for self medication was NSAIDS (non steroidal anti-inflammatory drugs) in both the groups being $75(100 \%)$. Antibiotics was self medicated by $26(2.66 \%)$ in group I and $14(18.66 \%)$ in group II.

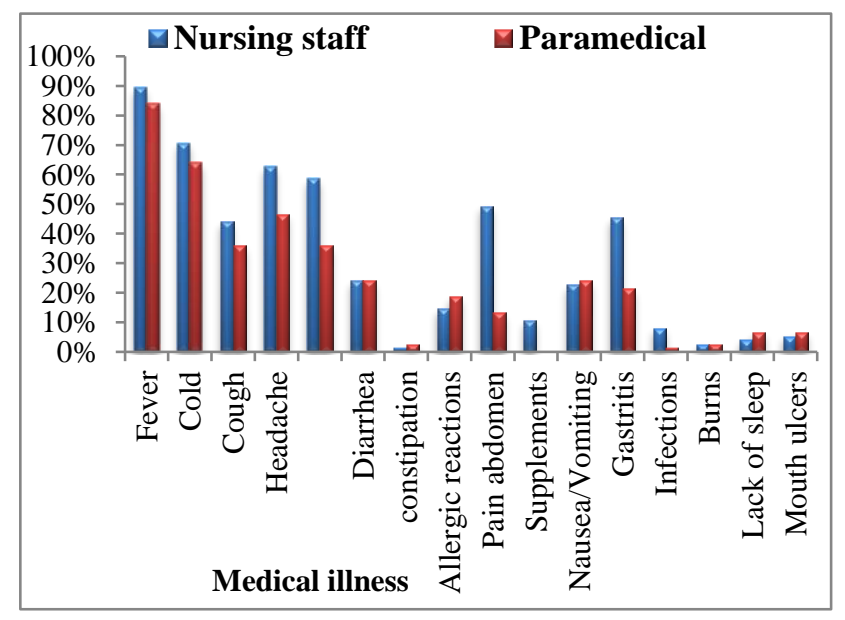

Figure 1: Medical illness for which self medication was practiced by the healthcare workers.

\section{DISCUSSION}

The overall knowledge, attitude and practice of self medication among the healthcare workers in the present study was encouraging. Healthcare workers gain adequate knowledge regarding medications and practice self medications which would influence the other populations with less knowledge related to medications. Other than the occupation related to health socioeconomic status, technologies such as internet, media etc promotes self medication in several populations. ${ }^{15}$

The overall prevalence of self medication in nursing staff and paramedical staff was $100 \%$ in this study were as in a study conducted by Williams A et al the prevalence of self medication among nursing and midwifery students was $91.7 \% .^{16}$ In a study conducted by Asa auta et al, the prevalence of self medication among medicine vendors was $75.4 \% .{ }^{17}$ Another study conducted among the health workers in tertiary hospital at Ondo State, Nigeria reported a prevalence of $73 \% .{ }^{18}$ Demographic variable such as education status, socioeconomic status, gender and age also affect the status of self medication, with Selfmedication being more in advanced education, in middle income earners, female and lesser than 40 years of age. ${ }^{19}$

Self medication is practiced due to the advantage of being convenient, saves time from consulting doctor, helps in treating minor ailments, being more economical when reaching doctor becomes difficult and early/initial relief of symptoms before consulting doctor. ${ }^{20,21}$ Though self medication has advantages it is becoming an occupational hazard due to the ill effects of self medications such as use of sub therapeutic or toxic doses, use of improper drug, drug interactions, drug abuse or drug dependance, failure to diagnose the illness, wastage of health resources due to illicit use and drug resistance. ${ }^{20,21}$ Self medication or irrational use of antibiotics has led society to antibiotic resistance which is a serious health problem worldwide. $^{22,23}$ 
The major factor which influence the Healthcare worker to practice self medication was by observing the drug prescribed by the practitioner constituting $35(46.7 \%)$ in nursing staff and $22(29.3 \%)$ in paramedical staff which was significant $(p=0.029)$. Palestinian medical and non medical students practiced self medication as the majority of them $(58 \%)$ felt the illness was simple and also from the experience of previous episodes $(29 \%) .{ }^{24}$ Media plays a highly influential role in procuring information related to self medications in general population. Compared to the other media internet carry more than $90.0 \%$ of the global information capacity. In this study group II preferred (22 $(29.3 \%)$ internet to gain knowledge related to self medication more compared to the group I. literacy and public health education also plays a vital role in practicing self medication. ${ }^{25}$

Nowadays there is an increase trend in the transfer of prescription ("Rx") medicines to non-prescription or OTC medicines which is known as "Rx-to-OTC switch". ${ }^{26}$ OTC switch occurs when a new medicine after being used in large population with adequate scientific information for suitable conditions manufacturer may choose to apply to procure OTC status from appropriate authority. ${ }^{26}$ Knowledge regarding the OTC medications which are the most common groups of drugs practiced for self medication was better in the nursing group than the other paramedical staff in this study. OTC drugs are effective and safer if used with appropriate instruction, information for proper diagnosis with adequate dosage and route of administration. Precautions should be laid when used in children, geriatric age group and pregnancy. ${ }^{27,28}$

In this study, the most common reasons reported by a large number of participants for self-medication was for common ailments such as fever [which was highest constituting 67(89.3\%) in nursing staff and 63(84.00\%)in other paramedical staff], cold, cough, headache, musculoskeletal pain etc (Figure 1). Residents in Wuhan, China practiced self medication for cough and cold the most $(55.1 \%) .{ }^{29}$ Due to the ease of procuring the medications from the hospital or the working place several drugs belonging to different groups was self medicated among the healthcare workers in this study. NSAIDS was the most common drug self medicated in all the participants, followed by antihistamine and drugs acting on GIT. Antibiotics were commonly used by the nursing staff $(34.66 \%)$ compared to the other paramedical staff. In a study conducted by Belachew Gutema $\mathrm{G}$ et al, reported NSAIDS (42.2\%) followed by antibiotics to be the most common group of drug self medicated by the health sciences students. ${ }^{30}$

Though self medication has advantages, self- medication has been negatively used as malpractice with increased risk of adverse drug reactions, addiction, drug dependence, drug interactions, food and drug interactions, inadequate dosing and polypharmacy. ${ }^{31}$ In this study $6.7 \%$ of non nursing staff practiced self medication to treat the side effects due to self medication. Such practices would lead to inadvertent effects of self medication and can harm ones health status, hence it is always appropriate to follow appropriate instructions before self medications and consult the practitioner to treat the adverse effects due to self medication.

\section{CONCLUSION}

Self care plays a vital role in maintenance of health. Healthcare workers having the knowledge of medicines prescribed through their work experiences are more prone to self medication and also influence the other non medical related population to practice self medication. It is always advisable to approach the physician for treating ailments when the self medication practice in not a responsible one.

\section{ACKNOWLEDGEMENTS}

The Authors of this study are highly thankful to the Healthcare workers of Mc.Gann Teaching hospital, SIMS, Shivamogga for their help and cooperation in conducting the present study.

\section{Funding: No funding sources}

Conflict of interest: None declared

Ethical approval: The study was approved by the Institutional Ethics Committee (SIMS/IEC/268/2015-16)

\section{REFERENCES}

1. WHO. WHO definition of Health. Off Record World Health Organisation [Internet]. 1948;(2):100. Available from: http://www.who.int/a

2. World Health Organisation RO for S-EA. Self care for health: A Handbook for Community Health Workers andamp; Volunteers [Internet]. Self Care for Health-A Handbook for Community Health Workers and Volunteers. 2013:7-15. Available at: http://apps.searo.who.int/PDS_DOCS/B5084.pdf

3. Ayalew MB. Self-medication practice in Ethiopia: A systematic review. Vol. 11, Patient Preference and Adherence; 2017:401-413.

4. Eldin SHN, ELKhawad AO. Self-medication with Antibiotics among Patients Attending Community Pharmacies in Khartoum City. Sudan J Ration Use Med [Internet]. 2014;(6):14-5. Available at: http://apps.who.int/medicinedocs/documents/s22205e n/s22205en.pdf

5. Goh LY, Vitry AI, Semple SJ, Esterman A, Luszcz MA. Self-medication with over-the-counter drugs and complementary medications in South Australia's elderly population. BMC Complement Altern Med. 2009;9:42.

6. Sherazi, B., Mahmood K. Prevalence and measure of selfmedication: A review. J Pharm Sci Res [Internet]. 2012;4(3):1774-8. Available at: https://pdfs.semanticscholar.org/a734/8fa949984d3a8 1a3919aeea7d8883f3eeb45.pdf

7. Galato D, Galafassi L de M, Alano GM, Trauthman SC. Responsible self-medication: review of the 
process of pharmaceutical attendance: Braz j pharm sci [Internet]. 2009;45(4):625-33. Available at: http://www.scielo.br/scielo.php?script=sci_arttextand pid=S1984-82502009000400004

8. World Self-medication Industry. Responsible SelfCare and self-medication - A worldwide review of consumer surveys. Wsmi [Internet]. 2006:1-16. Available at: http://www.wsmi.org/pdf/wsmibro3.pdf

9. Reza E, Davarani B, Mahmoudvand H, Jamshidi E, Jahani Y. Self-medication among health staff: A cross sectional study of Kerman. 2016;8(12):221-8.

10. Seam M, Bhatta R, Saha B, Das A, Hossain M, Uddin $\mathrm{S}$, et al. Assessing the Perceptions and Practice of SelfMedication among Bangladeshi Undergraduate Pharmacy Students. Pharmacy. 2018;6(1):6. Available at: http://www.mdpi.com/2226-4787/6/1/6.

11. Dineshkumar B, Raghuram TC, Radhaiah G, Krishnaswamy K. Profile of drug use in urban and rural India. Pharmacoeconomics. 1995;7:332-46.

12. Jain S, Malvi R, Purviya JK. Concept of Selfmedication; a Review. International Journal of Pharmaceutical and Biological Archives. 2011;2(3):831-6.

13. Ehigiator O, Azodo CC, Ehikhamenor EE. Selfmedication with antibiotics among Nigerian dental students. Tan Dent J. 2010;16(2):48-53.

14. Babatunde OA, Fadare JO, Ojo OJ, Durowade KA, Atoyebi OA, Ajayi PO, et al. Self-medication among health workers in a tertiary institution in South-West Nigeria. Pan African Med J. 2016;24:1-8.

15. Gyawali S, Shankar PR, Poudel PP, Saha A. Knowledge, Attitude and Practice of Self-Medication Among Basic Science Undergraduate Medical Students in a Medical School in Western Nepal. J Clin Diagn Res. 2015;9(12):FC17-22.

16. Williams A, Crawford K. Self-medication practices among undergraduate nursing and midwifery students in Australia: a cross-sectional study. Contemp Nurse. 2016;52(4):410-20.

17. Auta A, Omale S, Folorunsho TJ, David S, Banwat SB. Medicine vendors: Self-medication practices and medicine knowledge. N Am J Med Sci. 2012;4(1):248.

18. Afolabi AO, AkinmoledunVI, Adebose IJ, Elkwachi $\mathrm{G}$.Self- medication profile of dental patient in Ondo State, Nigeria. Nig J Med. 2010;19(1):96-103.

19. Ifedili CJA, Ifedili CA. Self-medication among Academicians in a Nigeria University. Are there gender and age differences? $\mathrm{J}$ of $\mathrm{Col}$ of Medic. 2008;13(1):29-33.

20. Sd V, Sneha A, Kamarudin J, Sm K. Self Medication - Reasons, Risks and Benefits. Inte $\mathrm{J}$ of Hea and Biomed Rese. 2016(04)):21-4.

21. Sarahroodi S. Self-medication: Risks and Benefits. International Journal of Pharmacol ogy. 2012;8(1):58-
9. Available at: http://www.scialert.net/abstract/?doi=ijp.2012.58.59

22. Al Rasheed A, Yagoub U, Alkhashan H, Abdelhay O, Alawwad A, Al Aboud A, et al. Prevalence and Predictors of Self-Medication with Antibiotics in $\mathrm{Al}$ Wazarat Health Center, Riyadh City, KSA. Biomed Res Int. Hindawi Publishing Corporation; 2016;2016.

23. Shah JJ, Ahmad H, Rehan BB, Najeeb S, Mumtaz M, Jilani $\mathrm{HH}$, et al. Self-medication with antibiotics among non-medical university students of Karachi: A cross-sectional study. BMC Pharmacology and Toxicology. 2014;15(1):1-7.

24. Sawalha AF. A descriptive study of self-medication practices among Palestinian medical and nonmedical university students. Res Soc Adm Pharm. 2008;4(2):164-72.

25. World self medication industry. The world federation promoting better health through responsible selfmedication NGO in official relations with the World Health Organization. Available at: http://www.wsmi.org/news/news_113.htm

26. Bennadi D. Self-medication: A current challenge. Journal Basic Clinical Pharmacology. 2014;5(1):19. Available at: http://www.jbclinpharm.org/text.asp?2014/5/1/19/12 8253

27. Subin MZ, Vidya V, Halima OA, Geethu G, Devika N. Monitoring the safety aspects of over the counter medications. Int Res J Pharm [Internet]. 2012;3(3):109-11.

28. Amoako EP, Richardson-Campbell L, KennedyMalone L. Self- medication with over-the-counter drugs among elderly adults. J Gerontol Nurs. 2003;29:10-5.

29. Lei X, Jiang H, Liu C, Ferrier A, Mugavin J. Selfmedication practice and associated factors among residents in Wuhan, China. Int J Environ Res Public Health. 2018;15(1).

30. Belachew GG, Alemayehu GD, Fikadu BD, Hadgu BA, Ghezu HM, Solomon HG, et al. Self-Medication Practices among Health Sciences Students: The Case of Mekelle University. J Appl Pharm Sci. 2011;1(2011):183-9.

31. Hussain A, Khanum A. Self-medication among university student of Islamabad, Pakistan; A preliminary study. Southern Med Review. 2008;1(1):14-6.

Cite this article as: Hanumaiah V, Harini M. Study of knowledge, attitude and practice of self medication among health care workers at MC Gann Teaching District hospital of Shivamogga, India. Int J Basic Clin Pharmacol 2018;7:1174-81. 